Un congreso para el humanismo:
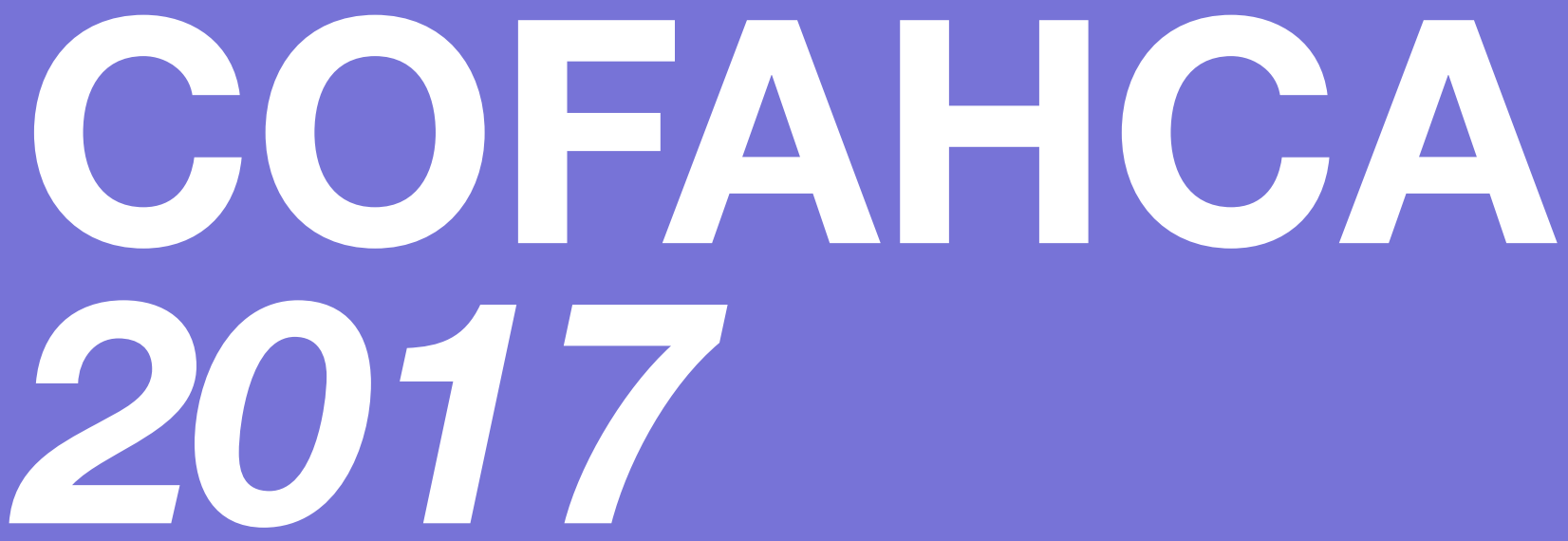

Ruth Nohemi Rojas Icabalzeta 


\section{Un congreso para el humanismo: COFAHCA 2017}

Nobemi Rojas Icabalzeta

Docente-Investigadora

Fac. de Humanidades y Ciencias Jurídicas Coord. de Comunicación para el Desarrollo UNAN-Managua ORCID. 0000-0003-4003-2288 nahomyrojas21@gmail.com

Copyright $(2017$ UNAN-Managua

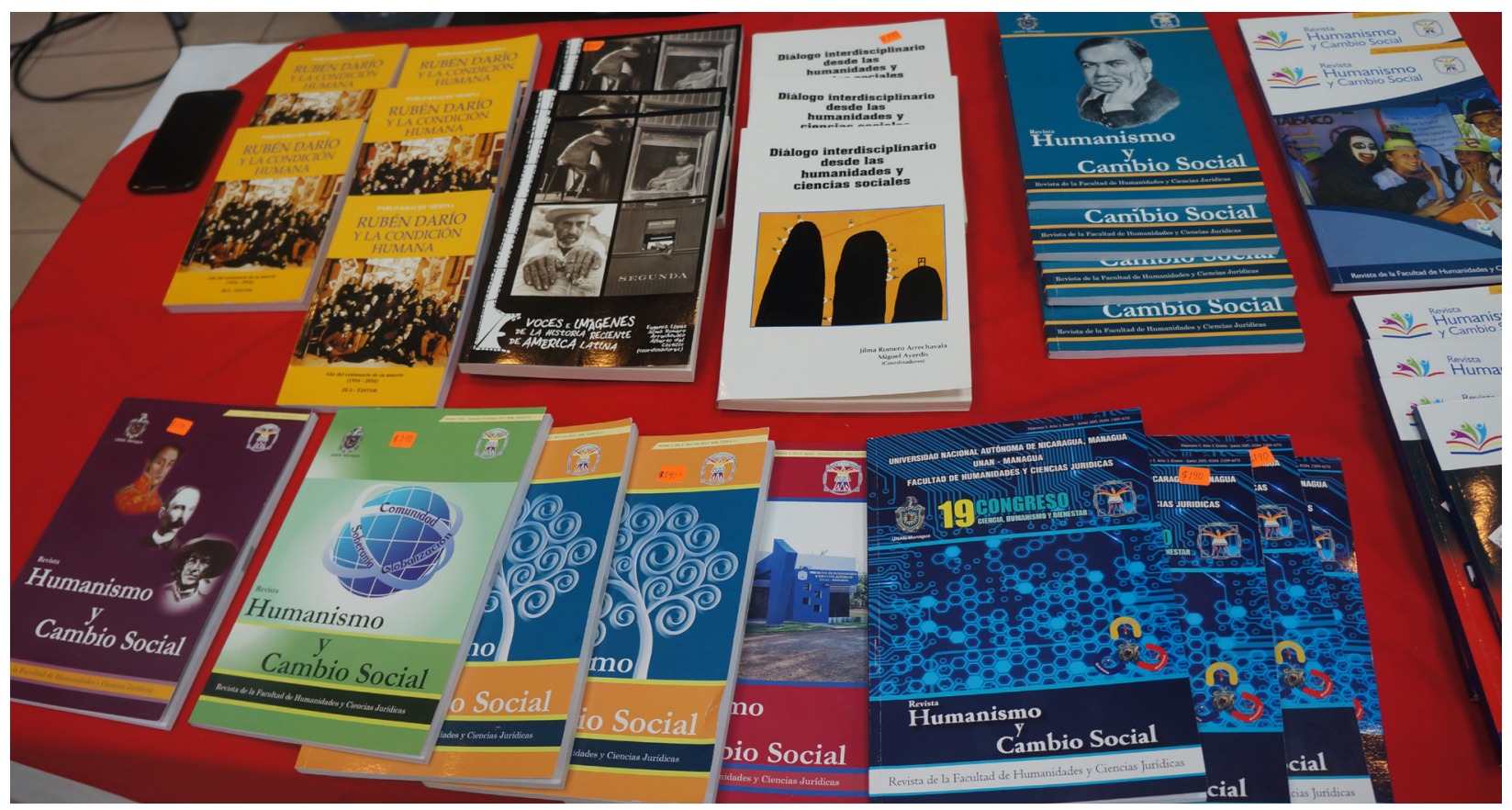

$\mathbf{E}$ I mundo actual demanda el ejercicio de la reflexión, el análisis críticoautocrítico y propositivo en aras de la humanización como elemento innato de los seres humanos. Estos aspectos que caracterizan el proceso de cada individuo fueron el cimiento del VIII Consejo de Facultades Humanísticas de Centroamérica y el Caribe (COFAHCA) 2017.

El VIII Congreso de Pensamiento Humanista Regional: El humanismo en la sociedad contemporánea tuvo como sede el Recinto Universitario Rubén Darío de la UNAN-Managua los días 26 y 27 de septiembre del año 2017. El evento se enmarca en los encuentros anuales de COFAHCA, que propicia un importante espacio de intercambio académico-investigativo.

El congreso reunió a representantes de facultades, sedes, direcciones y recintos de universidades de Guatemala, El Salvador, Honduras, Costa Rica, Panamá, República Dominicana, Puerto Rico y Nicaragua, quienes trabajan por la integración sobre las bases de la cultura y educación de los países de la región. 
Los objetivos del congreso se fundamentaron en promover el diálogo entre expertos investigadores y docentes en torno a los ejes temáticos desde las experiencias e innovaciones del quehacer universitario; abrir espacios para socializar y compartir experiencias dentro del campo del humanismo en los procesos de investigación y acción social para el avance en el desarrollo sociocultural de la región; y consolidar redes institucionales para el abordaje de estrategias de investigación que vinculen temáticas de las Humanidades en Centroamérica y el Caribe.

El evento académico se desarrolló como parte de las actividades conmemorativas del 18 de Septiembre, Día del Humanista, para rendir homenaje a los hermanos patriotas nicaragüenses Ángela y Ricardo Morales Avilés. Ricardo fue asesinado por la guardia somocista a los 34 años de edad, el 17 de septiembre de 1973, en el municipio de Nandaime. Fue un catedrático que se desempeñó como docente de Psicología de la UNAN, y se le considera un intelectual comprometido con sus valores e ideales de lucha por el bienestar de su pueblo.

\section{El contexto del COFAHCA}

El COFAHCA nació en 1993 como un organismo promotor, coordinador, ejecutor y difusor de políticas, programas, intercambios, proyectos, publicaciones y otras actividades de carácter académico, con énfasis en el elemento humanístico. Este se germinó a partir de la necesidad de destacar el pensamiento humanista regional en la labor formativa de los centros de educación superior. El Maestro Elmer Cisneros (q.e.p.d.), Rector de la UNAN-Managua (2010-2015), fue uno de sus fundadores.

Con más de dos décadas de existencia, el COFAHCA ha pasado de ser un pequeño grupo de visionarios a un sólido foro que ha tenido en su interior a representantes de facultades, sedes, direcciones y recintos de universidades de la región, incluidos Panamá, República Dominicana, Puerto Rico y México.

Desde el punto de vista de la gestión, COFAHCA es una instancia capaz de generar escenarios que no solo posibilitan espacios regionales donde intelectuales se reúnen para repensar las humanidades en la sociedad moderna, sino que también promueve líneas de acción para implementar políticas y directrices en materia educativa, científica y tecnológica, tanto en el ámbito de las universidades como en la sociedad en general.

De esta manera, el propósito de este consejo es vincular académicamente a las facultades de humanidades, pero sobre todo promover el humanismo en la formación de los estudiantes universitarios a nivel regional, mediante el debate de realidades y situaciones complejas que en la era global la educación superior debe enfrentar. 


\section{La experiencia de organización y sistematización}

La organización del VIII Congreso estuvo a cargo de la Facultad de Humanidades y Ciencias Jurídicas, representada por la Maestra Lidia Cortés Castillo, en ese momento Vicedecana, y un equipo de sistematizadores integrado por los maestros Xavier Rodríguez, Álvaro Muñoz González y Anabell Ibarra. Asimismo, alumnos ayudantes y personal administrativo colaboraron en la coordinación de mesas de trabajo, aseguramiento de la logística y en la organización de un tour para docentes invitados, a fin de integrarlos a la cultura nicaragüense.

El congreso permitió la interacción e intercambio de experiencias para el fortalecimiento de los ejes temáticos, los cuales se concentraron en la misión humanística con responsabilidad social para el desarrollo humano, el legado de los pensadores humanistas regionales, el humanismo en la sociedad del conocimiento y el humanismo en el escenario del quehacer de las universidades públicas.

De un total de 385 participantes entre estudiantes, investigadores y docentes de la región, se presentaron unas 25 ponencias que se distribuyeron en mesas de trabajo, cada una de las cuales contó con el apoyo de un coordinador, un moderador y un sistematizador. Estos fueron los responsables de garantizar el cumplimiento del programa que permitió un espacio de retroalimentación entre los ponentes y la comunidad universitaria participante. Además, se planificaron dos conferencias magistrales y la presentación de publicaciones de la UNAN-Managua.

Entre las instituciones académicas que participaron estuvieron la Universidad de Costa Rica, Universidad Nacional de Costa Rica, Universidad Autónoma de Chiriquí Panamá, Centro Regional Universitario de Veraguas de Panamá, Universidad de Panamá, Escuela de Formación de Profesores de Enseñanza Media de la Universidad de San Carlos, Guatemala, Universidad Tecnológica de El Salvador, Universidad Autónoma de Santo Domingo, Universidad Politécnica de Nicaragua, Universidad Nacional Autónoma de Nicaragua, León (UNAN-León) y Universidad Nacional Autónoma de Nicaragua, Managua (UNAN-Managua) en sus facultades de Humanidades y Ciencias Jurídicas, Educación e Idiomas, Ciencias e Ingeniería y la Facultad Regional Multidisciplinaria de Estelí (FAREM-Esteli). 


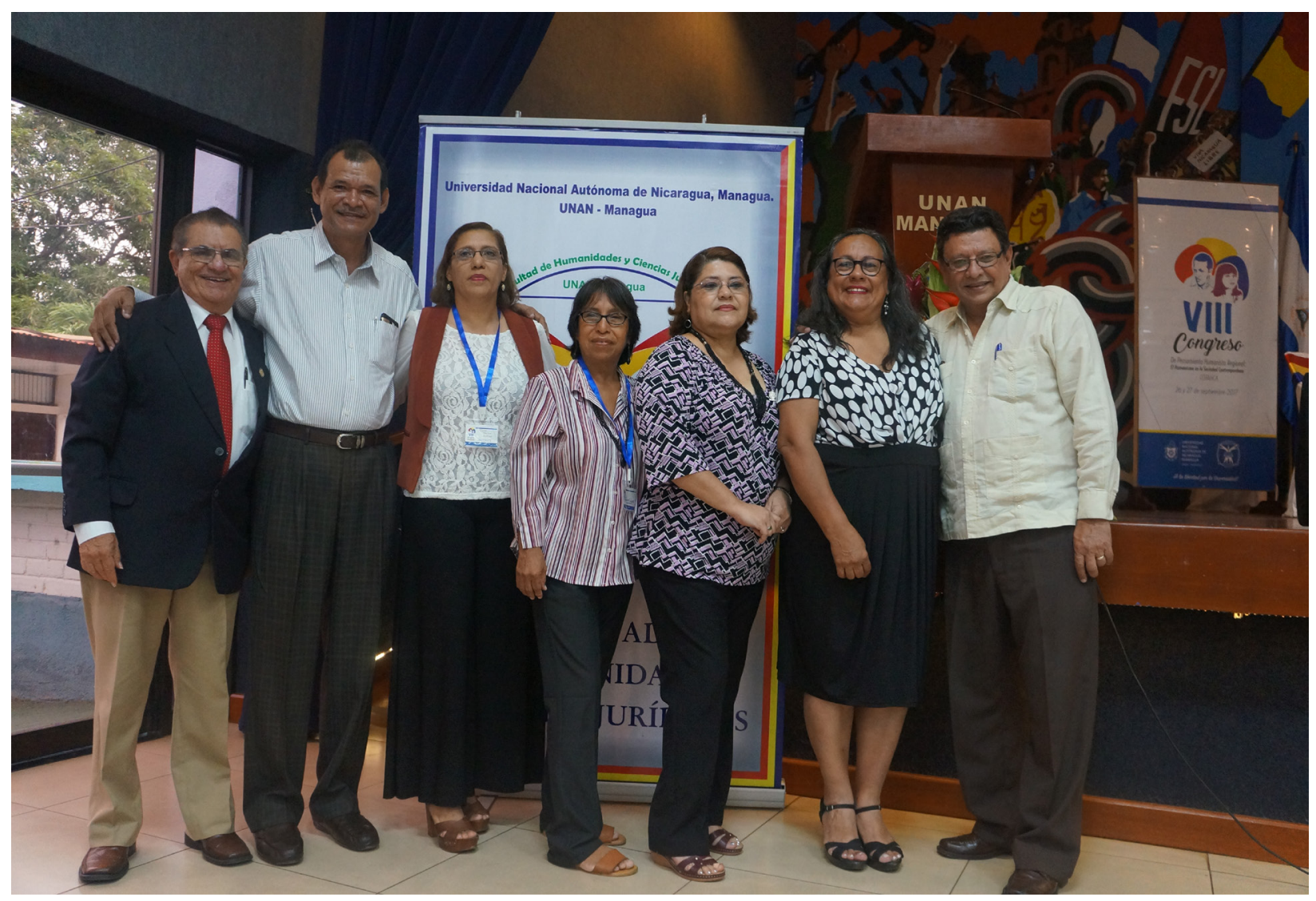

\section{Desafíos para la universidad}

En su afán por la búsqueda de más espacios acertados para los aprendizajes, el COFAHCA 2017 permitió a la UNAN-Managua plantearse algunos desafíos, entre estos la creación de programas de seguimiento para evaluar los resultados y alcances, tanto de los programas de asignatura orientados al humanismo como de las investigaciones derivadas de la praxis académica. De la misma manera, se vincula la necesidad de articular a la región centroamericana en un esfuerzo por la implementación de programas que pongan de relevancia el papel del humanismo en el bienestar de los pueblos, así como la promoción de las dinámicas cotidianas de la región para revisar la teoría y prácticas desde otros contextos.

En adición a esta coyuntura, la multiculturalidad e identidad y cosmovisiones de los pueblos centroamericanos también se concibieron como elementos dentro del proceso formativo humanista. En este sentido, se planteó que la universidad debe integrar el tema de los pueblos indígenas en las cátedras universitarias. La misma importancia retomó el tema de la interdisciplinariedad que se perfila como un desafío permanente para el que la universidad debe alcanzar niveles superiores.

Asimismo, en aras de construir espacios en común, durante el congreso se determinó la necesidad de compartir conocimientos a nivel local, regional y global derivado de las propias experiencias de la región. Para ello, se plantea la importancia de la interacción en los espacios académicos, con el fin de, entre otros logros, abonar al desarrollo de la cooperación sur-sur en el ámbito universitario. 


\section{Un humanismo integral para la formación universitaria}

EI COFAHCA 2017 fue el escenario propicio para debatir la creciente devaluación del humanismo ante la inversión de la jerarquía de los valores, la construcción de una filosofía del desarrollo y una ética basándose en el ser humano y la naturaleza en la búsqueda del macro y micro equilibrio entre ambos elementos. Ante tal contexto, la universidad se convierte en la instancia formadora de ciudadanos y profesionales que enfrentan el desafío del mundo actual para convertirlo en uno más humano donde se pueda vivir mejor.

La UNAN-Managua retoma el humanismo como una gama de oportunidades para que los académicos se inserten en los procesos sociales. Según la maestra Anabell lbarra, es apremiante que la universidad tenga mayor impacto y que las distintas ciencias y disciplinas confluyan en un proceso de investigaciones multidisciplinares e interdisciplinares que forjen el camino hacia el cambio social. Por su parte, el maestro Xavier Rodríguez considera que Nicaragua debe aprovechar este tipo de encuentros y con ello apostar a la integración de la región, puesto que aún hay muchos temas que se deben coordinar para desentrañar puntos en común.

De acuerdo con la Maestra Gloria López Alvarado, en en ese entonces Decana de la Facultad de Humanidades y Ciencias Jurídicas, el encuentro prescribe las humanidades para un desarrollo integral, desde la búsqueda de identificación de las raíces y quehaceres para ampliar el pensamiento humanista que ha sido una tarea constante en una alianza entre universidades públicas y no públicas.

La Maestra López Alvarado valora que el COFAHCA se fortalece desde las múltiples facetas de la vida social que implica que "la humanidad necesita humanismo y desde la academia esto se retoma en un sentido de interacción social humana". Añadió que el desarrollo humano se debe abordar no como desarrollismo, sino que en el imaginario individual y colectivo deben predominar la integración de temas como la equidad de género, salud integral, soberanía alimentaria y raíces de nuestras identidades, considerando el desarrollo humano como el componente de una condición de hegemonía de expresión horizontal.

El encuentro de COFAHCA permitió la releflexión puntual de que "la universidad no puede estar ajena a la revalorización humanística. De acuerdo con su misión y visión (el propósito) no es formar técnicos, tecnócratas ni profesionales esquemáticos, sino (profesionales) con una concepción científica, técnica y humanística, es decir con una formación integral”, lo cual realzaría el salto cualitativo para alcanzar un mundo posible y una humanidad más humana.

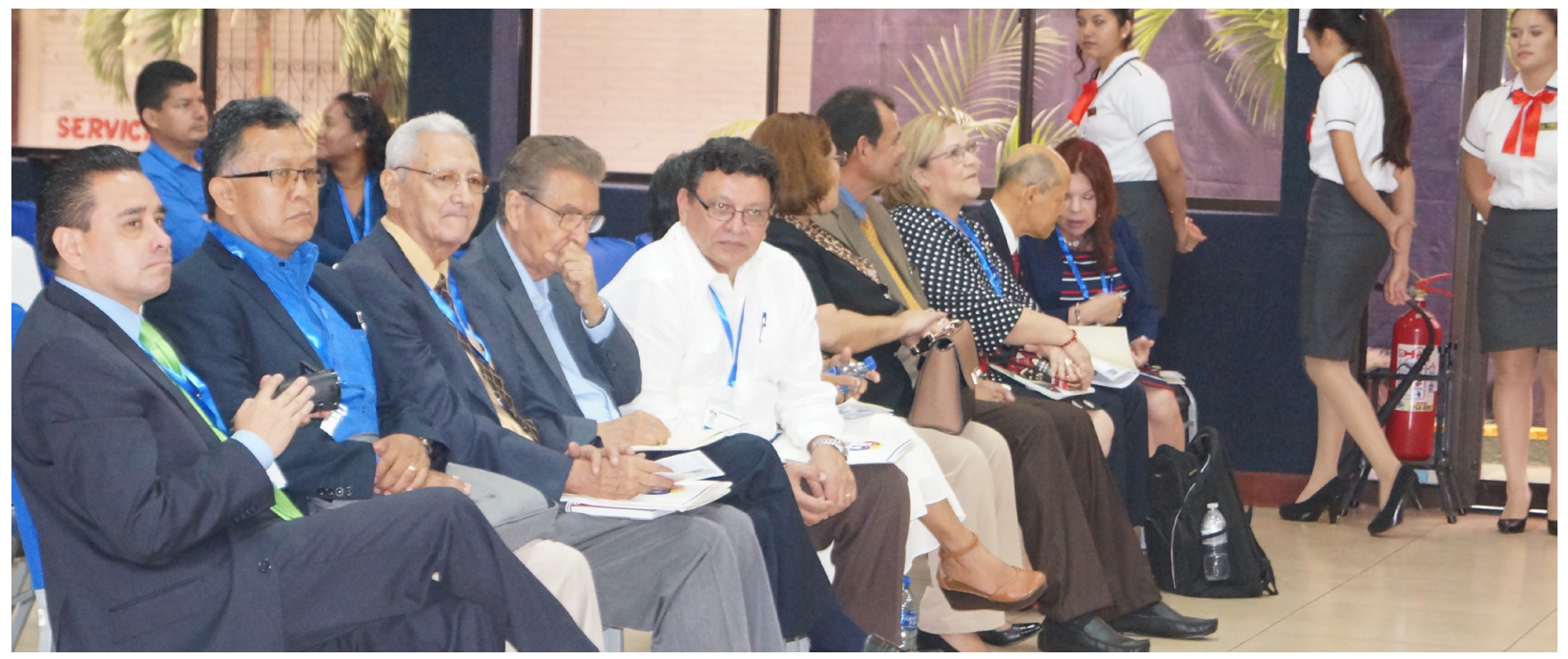

